\title{
Dromyosuppressin and Drosulfakinin, Two Structurally Related Drosophila Neuropeptides, Are Uniquely Expressed in the Adult Central Nervous System ${ }^{a}$
}

\author{
R. NICHOLS, ${ }^{b-d}$ J. MCCORMICK, AND I. LIM ${ }^{c}$ \\ Departments of Biological Chemistry" and Biology" \\ University of Michigan \\ Ann Arbor, Michigan 48109-1048
}

\section{INTRODUCTION}

Peptides present in the nervous system can act as hormones, transmitters, or modulators of various physiological functions. It is well established that more than one peptide messenger can exist in a single neuron and that neuropeptides can often be grouped into a family based on structure similarity. ${ }^{1}$ Although immunochemistry is a valuable technique in visualizing molecules present in a neuron, it is important to take into consideration the presence of structurally related peptides when designing antigens and interpreting immunocytochemical data. Antisera that distinguish between structurally related peptides are important experimental tools to determine expression and suggest function, as well as to analyze mutants and elucidate polypeptide precursor processing.

One family of neuropeptides is structurally related to the molluscan cardioexcitatory peptide FMRFamide ${ }^{2}$ via the C-terminus -XRFamide, where $\mathrm{X}=\mathrm{L}$ or M. ${ }^{3.4}$ Drosophila myosuppressin (TDVDHVFLRFamide; DMS) and sulfakinin (FDDYGHMRFamide; ${ }^{e}$ DSK) have structure similarity to FMRFamide. ${ }^{5.6}$ To study DMS and DSK expression, we have generated and characterized DMS- and DSK-specific antisera and performed double-label immunocytochemistry. Our results indicate that DMS and DSK are expressed in different neurons in the adult central nervous system.

\section{MATERIALS AND METHODS}

The antigens, TDVDHV-MAP and FDDYGH-MAP, where MAP represents multiple antigenic peptide, ${ }^{7}$ were designed to the variant $\mathrm{N}$-terminal sequences of DMS and DSK, respectively. Antisera were raised in New Zealand white rabbits

${ }^{a}$ This work was supported by an NSF grant (IBN No. 9409623) and an AHA/MI grant to R.N.

${ }^{d}$ Address correspondence to R. Nichols, $830 \mathrm{~N}$. University Street, University of Michigan. Ann Arbor, MI 48109-1048. E-mail: nicholsr@umich.edu

$\ell$ In FDOYGHMRFamide, the bold type $\mathbf{Y}$ represents a sulfated tyrosyl residue. 
and analyzed by indirect immunofluorescent analysis of whole-mount third-instar larval central nervous system tissue as previously described. ${ }^{8}$ Antisera were purified on peptide affinity columns made by coupling the antigen to Affi-gel 10 (BioRad Labs) according to the manufacturer's specifications. ${ }^{8}$ Affinity-purified antisera were characterized by preincubation with FDDYGHMRFamide or TDVDHVFLRFamide prior to immunocytochemistry.

Double-label immunochemistry was performed using a modified single-label method. ${ }^{8}$ Whole-mount tissue preparations were incubated with the first primary antisera for $4-6 \mathrm{~h}$. rinsed in $0.5 \mathrm{M}$ sodium phosphate, $\mathrm{pH} 7.2$, with $0.2 \%$ Triton $\mathrm{X}-100$ and $1 \%$ sodium azide (PTN), incubated in CY3-conjugated Fab fragment goat anti-rabbit secondary antibody (Jackson ImmunoResearch Labs, West Grove, PA) for 4-6 h, rinsed in PTN for $2 \mathrm{~h}$. incubated in the second primary antisera for 4-6 h, rinsed in PTN for $2 \mathrm{~h}$, and incubated in FITC-conjugated goat antirabbit secondary antibody (Sigma, St. Louis, MO) for 4-6 h. Tissue was then extensively washed and prepared for microscopy as previously described. ${ }^{8}$ Data. collected with a Bio-Rad MRC 600 laser scanning confocal microscope equipped with a $\mathrm{Kr}$-Ar laser attached to a Nikon inverted microscope, were processed with Adobe Photoshop and transferred to Kodak slide film using a Macintosh Quadra 800 and Lasergraphics LFR-X.

\section{RESULTS AND DISCUSSION}

Nomenclature used in describing the neuronal staining of DMS and DSK antisera is based on previous publications identifying FMRFamide-like immunoreactive materials. ${ }^{5.6 .9-11}$ Immunoreactivity was observed bilaterally symmetric to the midline such that reference to one neuron indicates that there were two neurons positioned bilaterally symmetric to one another. Signal intensity was strong and consistent, and no fewer than eight preparations were analyzed.

Antisera to FMRFamide have been used to stain Drosophila neural tissue $;^{5-11}$ however, given the number of FMRFamide-like peptides and that antisera to FMRFamide recognize the common C-terminal structure, the data are ambiguous and the expression patterns of individual FMRFamide-like peptides cannot be identified. To study the expression of peptides structurally related to FMRFamide, we generated antisera to multiple antigenic peptides designed to the variant $\mathrm{N}$ terminal sequences. We chose to use MAP antigens because of the advantages that they offer including the fact that no carrier molecule is required and that multiple copies of the antigen are present in a single molecule. ${ }^{7}$

To study whether FMRFamide-like peptides coexist we established a doublelabel immunofluorescence protocol using antisera raised in different animals of the same species host animal. Generation of antisera in the same species can be advantageous in terms of technical aspects such as animal housing and blood collection.

We have reported the individual expression patterns of both DMS and DSK immunoreactive materials during development using single-label immunocytochemistry. ${ }^{x .12}$ The neurons stained by each antisera were identificd based on

position, comparing the staining patterns with those of FMRFamide antisera. ${ }^{9-11}$ Although the single-label immunochemical data are informative, they cannot unequivocally answer the question of whether these peptides are expressed in the same neuron or in neurons in close proximity; it would be more definitive to compare DMS and DSK staining patterns within the same preparation. 


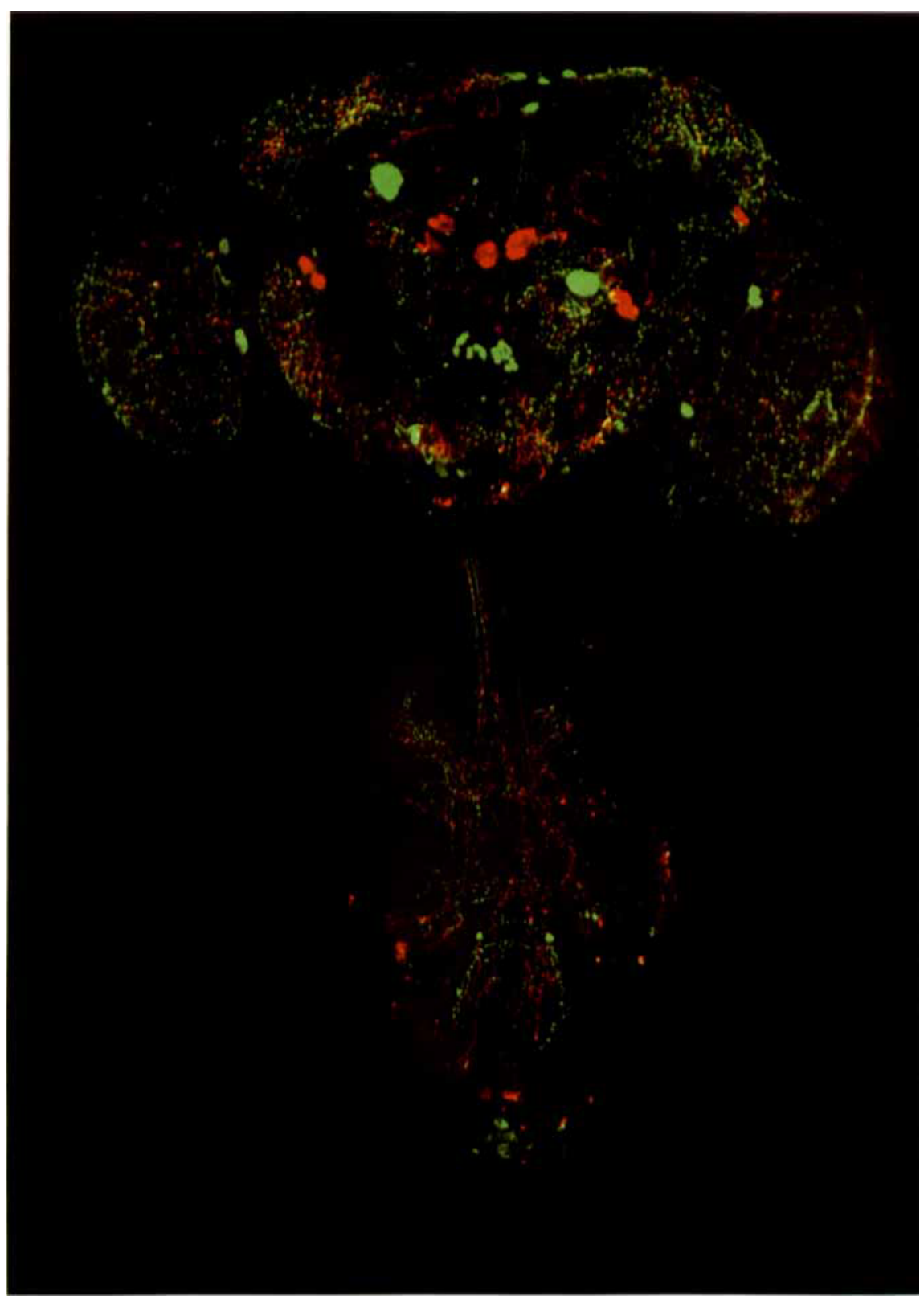

FIGURE 1. Double-label immunofluorescence of DMS and DSK in an adult central nervous system. DMS antisera are recognized by FITC-labeled (green) secondary antibody, whereas DSK antisera are recognized by CY3-labeled (red) secondary antibody. No neuron is stained by both antisera (yellow); some fibers appear yellow because the method of data collection results in overlaying neuronal projections that exist at different focal planes throughout the tissue. 
We have previously presented double-label immunofiuorescence data describing the expression patterns of DMS and DSK in larval neural tissue. ${ }^{13}$ In this paper we describe a more versatile double-label immunofluorescence protocol than used to study expression in larva and determine the DMS and DSK staining patterns in adult neural tissue. Although DMS- and DSK-specific antisera stain several neurons in the adult Drosophila central nervous system (FIG. 1), the expression patterns in the adult central nervous system are unique and nonoverlapping. Numerous neurons in close proximity to one another are stained by either DMS- or DSK-specific antisera, for example, DMS-specific antisera stain a MP2 neuron, whereas DSK-specific antisera stain two MPI neurons; however, no neuron is stained by both antisera, indicating that these structurally related neuropeptides do not coexist. The nonoverlapping expression patterns for DMS and DSK suggest that these peptides have different roles in the central nervous system.

\section{SUMMARY}

Drosophila myosuppressin (TDVDHVFLRFamide; DMS) and sulfakinin (FDDYGHMRFamide: DSK) have similar C-terminal structures. To determine the neuronal expression patterns of these structurally related peptides, we have generated DMS- and DSK-specific antisera to multiple antigenic peptides and performed double-label immunochemistry with antisera raised in different animals of the same species host animal. Our data indicate that DMS and DSK staining patterns in the adult central nervous system are unique and nonoverlapping.

\section{REFERENCES}

1. Hökfelt, T., V. R. Holets, W. Staines. B. Meister, T. Mel.ander, M. Schalling, M. Schultzberg, J. Frfedman, H. Björklund, L. Olson, B. Lindh, L.-G. Elffin, J. M. Lundberg. J. A. Lindgeren. B. Samuelsson, B. Pernow, L. Terenius. C. Post. B. Everitt \& M. Goldstein. 1986. In Progress in Brain Research. T. Hökfeit. K. Fuxe \& B. Pernow. Eds. Vol. 68: 33-70. Elsevier Science Ltd. Cambridge, UK.

2. Price, D. A. \& M. J. Greenderg. 1977. Science 197: 670-672.

3. RAFFA, R. B. 1988. Peptides 9: 915-922.

4. Price. D. A. \& M. J. Greenberg. 1989. Biol. Bull. 177: 198-205.

5. Nichols, R. 1992. Mol. Cell. Neurosci. 3: 342-347.

6. Nichols. R. 1992. J. Mol. Neurosci. 3: 213-218.

7. Posnett, D. N. \& J. P. Tam. 1989. In Methods in Enzymology. J. J. Langone, Ed. Vol. 179: 739-746. Academic Press. New York.

8. MCCormick, J. \& R. Nichols. 1993. J. Comp. Neurol. 338: 272-288.

9. White, K.. P. Hurteau \& P. Pusnal. 1986. J. Comp. Neurol. 247: 430-438.

10. Chin. A. C.. E. R. Reynolds \& R. Scheller. 1990. DNA Cell Biol. 9: 263-271.

11. Schneider. L. E.. M. A. O Brien \& P. H. Taghert. 1991. J. Comp. Neurol. 304: $608-622$.

12. Nichols. R. \& I. Lim. 1996. Cell Tissue Res. 283: 107-116.

13. TibBetts. M. F. \& R. Nichol.S. 1993. Neuropeptides 24: 321-325. 